\title{
Determination of closure domain penetration in electrodeposited microtubes by combined magnetic force microscopy and giant magneto-impedance techniques
}

\author{
J. M. Garcia, A. Asenjo, ${ }^{\text {a) }}$ and M. Vázquez \\ Instituto de Magnetismo Aplicado (UCM-RENFE)-Instituto de Ciencia de Materiales de Madrid (CSIC), \\ P.O. Box 155, Las Rozas 28230, Madrid, Spain \\ A. M. Yakunin and A. S. Antonov \\ Scientific Center for Applied Problems in Electrodynamics, 127412 Moscow, Russia \\ J. P. Sinnecker \\ Instituto de Fisica, Universidade Federal do Rio de Janeiro C.P. 68528, 21945-970 Rio de Janeiro, Brasil
}

(Received 20 March 2000; accepted for publication 7 December 2000)

The domain structure of electrodeposited $\mathrm{Co}_{90} \mathrm{P}_{10}$ microtubes exhibiting radial magnetic anisotropy and giant magneto-impedance effect has been characterized by combined magnetic force microscopy imaging and impedance measurements. It has been shown that the size of the closure domains increases with the CoP layer thickness. Furthermore, the depth of the closure domains has been quantitatively determined from the high frequency behavior. (C) 2001 American Institute of Physics. [DOI: 10.1063/1.1346998]

\section{INTRODUCTION}

The search for magnetic materials exhibiting new transport properties is a matter of the utmost importance from a technological point of view to develop novel sensors and actuators. Nowadays, particular interest is being devoted to the giant magneto-impedance effect (GMI) observed in different families of very soft magnetic materials. It consists of the very large change in the impedance due to a dc applied magnetic field. ${ }^{1,2}$ Recently, GMI has been observed in magnetic microtubes, i.e., heterogeneous samples which consist of nonmagnetic wires electroplated with magnetic layers exhibiting circular, ${ }^{3,4}$ helical $^{5}$ or radial $^{6}$ magnetic anisotropy. The origin of GMI is ascribed to the skin effect by which high frequency current is restricted to a very thin film near the surface of the conducting materials. ${ }^{7}$ Changes in the impedance represent a unique tool to investigate the magnetic properties near the surface of magnetic samples as the penetration depth depends on applied dc field via the transverse susceptibility. In particular, magnetic coatings on nonmagnetic metallic wires are attracting much attention for their applications as GMI sensing elements in different devices. ${ }^{8}$

Full technological exploitation of materials exhibiting GMI requires a deeper understanding not only of their transport phenomena but also of their magnetization behavior at a basic level. In this sense, magnetic force microscopy $(\mathrm{MFM})^{9,10}$ is a suitable tool for the proper characterization of such novel magnetic materials, and in particular of their surface, because of its very high lateral resolution on mapping the magnetic structure with minimal sample preparation. The main part of this work is the combination of MFM imaging and high frequency measurements of impedance in the $\mathrm{MHz}$ range to characterize the domain structure near the surface of

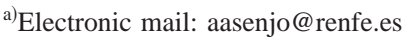

electrodeposited $\mathrm{Co}_{90} \mathrm{P}_{10}$ microtubes which exhibit radial magnetic anisotropy and GMI effect. It is shown that quantitative values of the size and depth of the closure domains can be deduced from these studies.

\section{ABOUT COP AMORPHOUS MAGNETIC MICROTUBES}

$\mathrm{CoP}$ amorphous magnetic microtubes have been prepared by means of the electrodeposition technique as described elsewhere. ${ }^{6} \mathrm{Co}_{90} \mathrm{P}_{10}$ thin layers with thickness ranging from 3 to $20 \mu \mathrm{m}$ have been electrodeposited onto $\mathrm{Cu}$ wires (diameter: $200 \mu \mathrm{m}$ ). Electrodeposited CoP amorphous microtubes exhibit magnetic anisotropy that depends on their thickness. ${ }^{11,12}$ The anisotropy of several $\mu \mathrm{m}$ deposited CoP alloy is mainly perpendicular to the surface as a consequence of the columnar growth of Co. Taking into account the cylindrical geometry of the substrates used here (Cu wires), the out-of-plane anisotropy leads to an easy axis in the radial direction. This characteristic has been verified by measuring the longitudinal hysteresis loops of the samples, whose shape indicates that the samples certainly exhibit an easy axis which is perpendicular to the axis of the tube. An anisotropy field of about $6 \mathrm{kA} / \mathrm{m}$ has been measured in all the samples. In spite of its perpendicular anisotropy, all these samples exhibit GMI effect as has been already reported..$^{6,13}$

\section{MAGNETIC FORCE MICROSCOPY MEASUREMENTS}

In order to analyze the domain structure of the $\mathrm{CoP}$ microtubes, MFM experiments have been carried out on the lateral surface of the microtubes using a commercial microscope from Nanotec ${ }^{\mathrm{TM}}$ operating in noncontact mode. The signal from the mechanically modulated cantilever is analyzed with the help of a lock-in amplifier and the force gra- 
a)

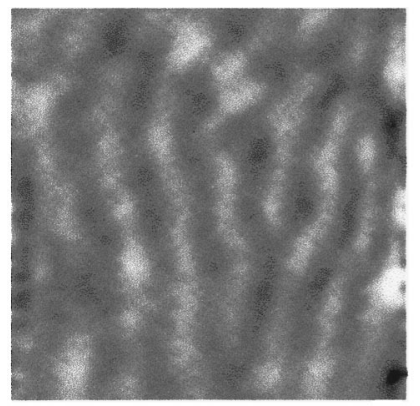

b)

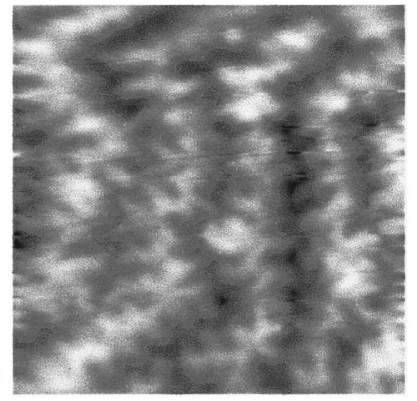

FIG. 1. MFM images (size: $28 \mu \mathrm{m} \times 28 \mu \mathrm{m}$ ) of $\mathrm{Co}_{90} \mathrm{P}_{10}$ microtubes with a thickness of $4 \mu \mathrm{m}$ (a), and $20 \mu \mathrm{m}$ (b).

dient is deduced from the phase shift of the signal. Both topographic and magnetic images are obtained simultaneously. Co-covered commercial tips from Park Scientific Instruments (resonant frequency $\sim 90 \mathrm{kHz}$, spring constant $\sim 0.5 \mathrm{~N} / \mathrm{m}$ ) magnetized along their pyramid axis have been employed. Further details about the measurement technique are reported elsewhere. ${ }^{14,15}$ All the MFM experiments have been performed in the remanence state of the samples.

The MFM images of the thinnest samples show stripe domains [see Fig. 1(a)] typical for materials exhibiting an out-of-plane anisotropy, with dark and bright areas corresponding to domains with up and down magnetization, respectively. As first considered by Kittel, ${ }^{16}$ the domain structure should provide flux closure within the film since the anisotropy field is much smaller than the demagnetizing field. However, no direct evidence of the existence of closure domains with in-plane orientation of the magnetization can be deduced from the MFM images of the thinnest samples. This fact can be ascribed to the small volume of the closure domains if the size of such closure structures is not large enough. The MFM magnetic signal emerging from these areas is smaller than the one rising from the main domains with perpendicular magnetization. Consequently, the closure domains cannot be detected by analyzing only the MFM images of the thinnest samples.

When the CoP layer thickness reaches $7 \mu \mathrm{m}$ and above, an undulation of the stripe domains is observed [Fig. 1(b)] so that the magnetostatic energy due to the presence of free poles on the sample surface is reduced. In addition, an increase in the domain width is observed. In such a case, main and closure domains can be distinguished in MFM experiments performed with a magnetic tip allowing high spatial resolution (tip radius of about $10 \mathrm{~nm}$ ), i.e., domains with perpendicular and in-plane magnetization, respectively [Fig. 2(a)]. It is important to mention that the image shown in Fig. 2(a) has been obtained using a small tip-to-sample distance (about $25 \mathrm{~nm}$ ). Consequently, some features of the image are induced by the strong magnetic interaction between the MFM tip and the sample. ${ }^{17}$ In particular, in the gray scale used, bright contrast is ascribed to an attractive interaction between tip and sample, whereas dark contrast corresponds to a repulsive interaction. Thus, the asymmetric contrast observed in the closure domain region, corresponding to an additional density of poles at the surface, appears as a con- a)

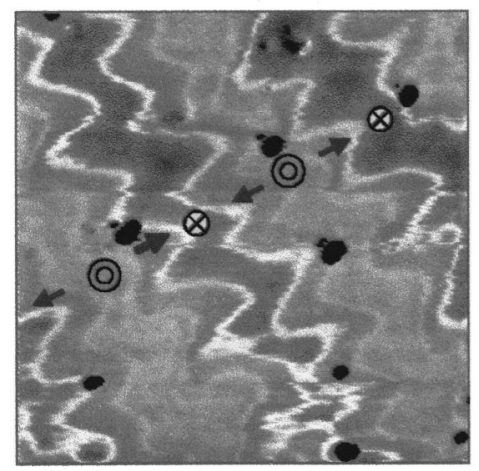

b)

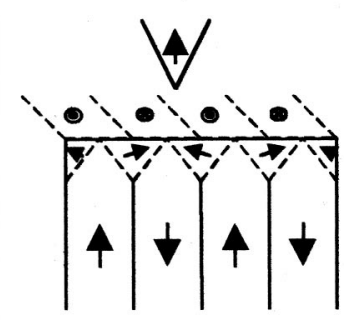

FIG. 2. MFM image (size: $12.5 \mu \mathrm{m} \times 12.5 \mu \mathrm{m}$ ) of a $\mathrm{Co}_{90} \mathrm{P}_{10}$ microtube with a $10 \mu \mathrm{m}$ thickness (a). Scheme of the MFM tip influence on the flux closure domain structure (b).

sequence of the tilting of magnetization in the closure domains towards the tip magnetization direction, as shown schematically in Fig. 2(b). Thus, by means of MFM imaging the existence of main domains together with closure domain structure has been detected in $\mathrm{CoP}$ microtubes thicker than 7 $\mu \mathrm{m}$. The size of the closure domains increases with the CoP layer thickness, and above $7 \mu \mathrm{m}$, gives rise to a magnetic signal which is strong enough to be detected using MFM.

\section{HIGH FREQUENCY IMPEDANCE MEASUREMENTS}

Valuable information of the closure domains and in particular of their size has been additionally estimated from impedance measurements in the $\mathrm{MHz}$ frequency range. These measurements have been performed using a coaxial transmission line whose inner conductor has been replaced by the magnetic sample. ${ }^{18}$ A detailed description of the measuring technique can be found elsewhere. ${ }^{19}$ Figures 3(a) and 3(b) show the frequency dependence of both real and imaginary components of the impedance $(Z=R-j X)$ at zero external field for two microtubes with CoP layer thickness of 3 and $20 \mu \mathrm{m}$, respectively. This frequency dependence of impedance is governed by the classical skin effect. ${ }^{20}$

As previously described, ${ }^{19}$ the magnetic permeability of the samples can be deduced from the measured impedance a)

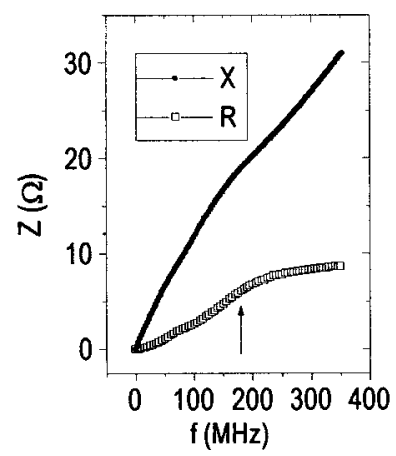

b)

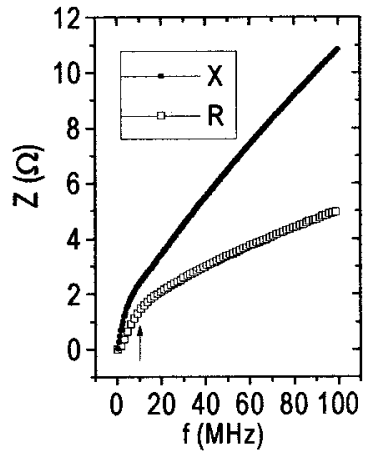

FIG. 3. Frequency dependence of both real (R) and imaginary (X) components of the impedance of $\mathrm{Co}_{90} \mathrm{P}_{10}$ microtubes with thickness of $3 \mu \mathrm{m}$ (a), and $20 \mu \mathrm{m}(\mathrm{b})$. 
a)

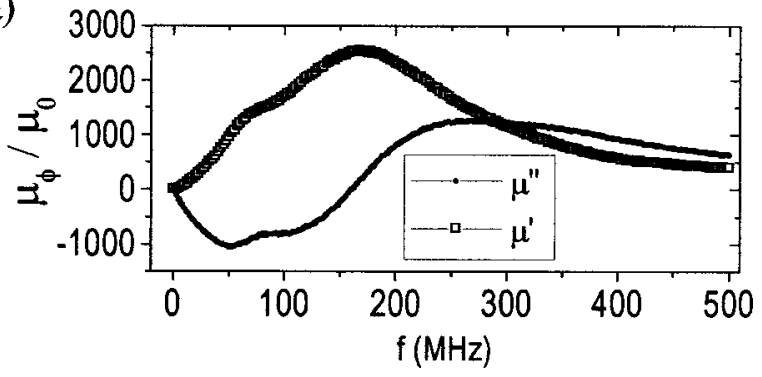

b)

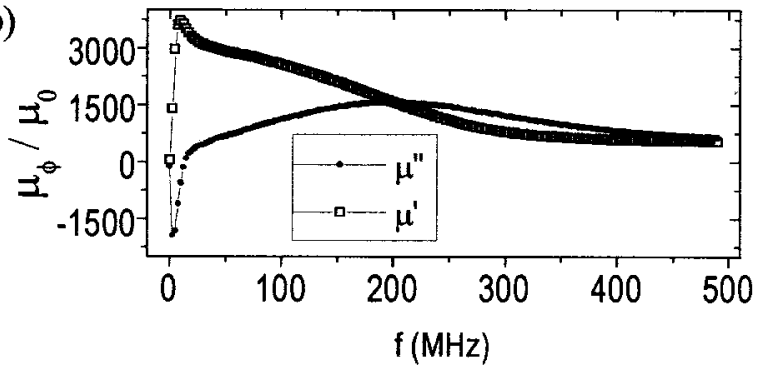

FIG. 4. Frequency dependence of both real $\left(\mu^{\prime}\right)$ and imaginary $\left(\mu^{\prime \prime}\right)$ components of the permeability of $\mathrm{Co}_{90} \mathrm{P}_{10}$ microtubes with thickness of $3 \mu \mathrm{m}$ (a), and $20 \mu \mathrm{m}$ (b).

assuming transverse electro-magnetic-mode propagation in the coaxial line, strong skin effect, and that the sample behaves as a single layered system. Under these conditions, the correlation between impedance, $Z$, and the propagation constant, $k$, is given by ${ }^{17}$

$$
Z=-i(2 / \omega) k \log (b / a) \tan (k l)
$$

for given geometrical factors ( $a$ and $b$ being the diameter of the sample and of the coaxial cell, respectively, and $l$ the length of the microtube) and angular frequency, $\omega$. The propagation constant is expressed as

$$
k^{2}=(\omega / c)^{2}[1+(1+j) / 2](\delta / a) \mu / \log (b / a)
$$

with $k=(1+i) / \delta$, and the skin depth $\delta=c /(2 \pi \sigma \omega \mu)^{1 / 2}, \sigma$ and $\mu$ being the conductivity and transverse permeability of the material, respectively.

Thus, by plotting the impedance as a function of the frequency, information is gained about the permeability of the effective radial region contributing to the impedance. In other words, when the slope of the impedance versus frequency curve changes significantly as frequency is lowered, it may be assumed that a new region with a significantly different magnetic permeability is now involved in the skin effect. Figure 4 shows the frequency evolution of both real and imaginary components of the permeability deduced from Eqs. (1) and (2) for two microtubes with different CoP layer thickness: $3 \mu \mathrm{m}$ [Fig. 4(a)] and $20 \mu \mathrm{m}$ [Fig. 4(b)].

Careful examination of Fig. 3(a) suggests a critical frequency of around $180 \mathrm{MHz}$ for the 3- $\mu$ m-thick microtube can be deduced. At frequencies higher than $180 \mathrm{MHz}$, the skin depth is so small that only the closure domain regions with mainly in-plane magnetization contribute to the measured impedance. However, when the frequency is lower than $180 \mathrm{MHz}$, the skin depth is larger than the thickness of the closure domain structures and the sample behaves as a two layer system: an external layer with in-plane magnetization (the closure domains) and an inner layer with out-ofplane magnetization (the main radial domains). As a consequence, at the critical frequency, and above, the single layer model can be applied, whereas at lower frequencies such model does not hold, i.e., the real component of permeability falls to zero and the imaginary component acquires negative values as shown in Fig. 4. Thus, the skin depth at the critical frequency where the above mentioned change of slope occurs denotes the depth of the closure domains. At some frequency below that transition the skin depth should reach the copper so actually the sample would become a three layer system and a new change of the slope for the impedance versus frequency curve should be observed. That additional transition frequency is not clearly observed in our case. According to a recent work, ${ }^{21}$ this transition takes place at $0.5-1 \mathrm{MHz}$.

Introducing the values of $\mathrm{CoP}$ microtube resistivity ( $\rho$ $\left.=9 \times 10^{-7} \Omega \mathrm{m}\right)$, critical frequency $(f=180 \mathrm{MHz})$ and permeability at this frequency $\left(\mu=2600 \mu_{0}\right)$ into Eq. (3) results in a depth of closure domain of $0.7 \mu \mathrm{m}$ for the $3-\mu \mathrm{m}$-thick microtube. In the case of the $20-\mu \mathrm{m}$-thick microtube, a similar analysis can be considered. From Fig. 3(b) a critical frequency around $10 \mathrm{MHz}$ is now deduced: only the closure domain regions contribute to the measured impedance above $10 \mathrm{MHz}$, whereas the main domains with perpendicular magnetization play the determinant role below $10 \mathrm{MHz}$. Equation (3) for a critical frequency of $10 \mathrm{MHz}$ and a reduced permeability at this frequency of 3800 results in $2.4-\mu$ m-deep closure domains for the $20-\mu \mathrm{m}$-thick microtube. Therefore, as expected from the MFM experiments, the size of the closure domains of the $20-\mu \mathrm{m}$-thick microtube is larger than that of the 3- $\mu \mathrm{m}$-thick microtube (2.4 and $0.7 \mu \mathrm{m}$, respectively).

\section{CONCLUSIONS}

In summary, the domain structure of $\mathrm{Co}_{90} \mathrm{P}_{10}$ electrodeposited microtubes exhibiting radial anisotropy and GMI effect has been determined using combined MFM imaging and impedance measurements in the $\mathrm{MHz}$ range. The existence of closure domain structures has been first observed by MFM images that allow us to conclude that the size of such domains increases with the CoP layer thickness. In addition, the impedance measurements have allowed us to calculate quantitatively the depth of such closure domains.

\section{ACKNOWLEDGMENTS}

The authors want to thank Professor J. Miltat for helpful discussions. This work has been performed under Project No. CAM/07N/0033/1998. A. Asenjo would like to thank the CAM (Spain) for the postdoctoral fellowship. J. P. Sinnecker thanks the Brazilian agencies CNPq and FAPERJ for the financial support.

${ }^{1}$ K. Mohri, T. Kohzawa, K. Kawashima, H. Yoshida, and L. V. Panina, IEEE Trans. Magn. 28, 3150 (1992).

${ }^{2}$ R. S. Beach and A. E. Berkowitz, Appl. Phys. Lett. 64, 3652 (1994).

${ }^{3}$ R. S. Beach, N. Smith, C. L. Platt, F. Jeffers, and A. E. Berkowitz, Appl. Phys. Lett. 68, 2753 (1996). 
${ }^{4}$ N. Usov, A. Antonov, and A. Granovsky, J. Magn. Magn. Mater. 171, 64 (1997).

${ }^{5}$ C. Favieres, C. Aroca, M. C. Sánchez, K. V. Rao, and V. Madurga, J. Magn. Magn. Mater. 196, 197, 224 (1999).

${ }^{6}$ J. P. Sinnecker, J. M. Garcia, A. Asenjo, and M. Vazquez, J. Mater. Res. 15, 751 (2000).

${ }^{7}$ L. V. Panina, K. Mohri, K. Bushida, and M. Noda, J. Appl. Phys. 76, 6198 (1994).

${ }^{8}$ M. Vázquez, J. Magn. Magn. Mater. (in press).

${ }^{9}$ Y. Martin and H. K. Wickramasinghe, Appl. Phys. Lett. 50, 1455 (1987).

${ }^{10}$ J. J. Sáenz, N. García, P. Grutter, E. Meyer, H. Heinzelmann, R. Wiesendanger, L. Rosenthaler, H. R. Hidber, and H. J. Guntherodt, J. Appl. Phys. 62, 4293 (1987).

${ }^{11}$ G. S. Cargill III, R. J. Gambino, and J. J. Cuomo, IEEE Trans. Magn. MAG-10, 803 (1974)

${ }^{12}$ J. M. Riveiro and M. C. Sanchez-Trujillo, IEEE Trans. Magn. MAG-16, 1426 (1980).

${ }^{13}$ M. Vázquez, J. M. García-Beneytez, J. M. García, J. P. Sinnecker, and A. P. Zhukov, J. Appl. Phys. 88, 6501 (2000).
${ }^{14}$ A. Asenjo, J. M. Garcia, D. Garcia, A. Hernando, M. Vazquez, P. A. Caro, D. Ravelosona, A. Cebollada, and F. Briones, J. Magn. Magn. Mater. 196, 197, 23 (1999).

${ }^{15}$ A. Asenjo, D. García, J. M. García, C. Prados, and M. Vázquez, Phys. Rev. B 62, 6538 (2000).

${ }^{16}$ C. Kittel, Phys. Rev. 70, 965 (1946).

${ }^{17}$ S. Foss, E. Dan Dahlberg, R. Proksch, and B. M. Moskowitz, J. Appl. Phys. 81, 5032 (1997).

${ }^{18}$ D. Menard, M. Britel, P. Ciureanu, A. Yelon, V. P. Paramonov, A. S. Antonov, P. Rudkowski, and J. O. Strom-Olsen, J. Appl. Phys. 81, 4032 (1997).

${ }^{19}$ A. Antonov, A. Granovsky, A. Lagar'kov, N. Perov, N. Usov, and T. Furmanova, Physica A 241, 420 (1997).

${ }^{20}$ L. D. Landau, E. M. Lifschitz, and L. P. Pitaevskii, Electrodynamics of Continuous Media (Butterworth-Heinemann, London, 1995), p. 212.

${ }^{21}$ J. P. Sinnecker, M. Knobel, R. Pirota, J. M. García, A. Asenjo, and M. Vázquez, J. Appl. Phys. 87, 4825 (2000). 